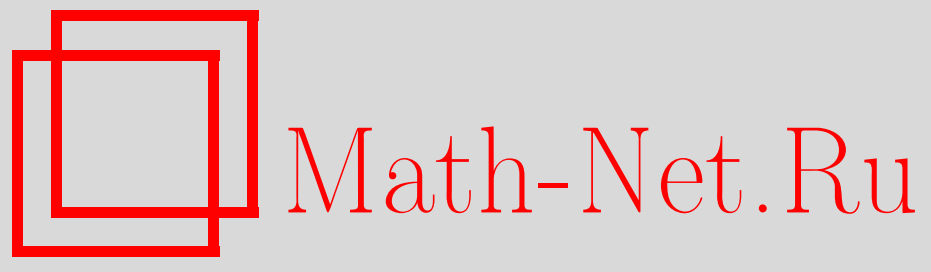

А. М. Райгородский, К одной теореме Ловаса о хроматическом числе сферы, Матем. заметки, 2015, том 98, выпуск 3, 470-471

DOI: https://doi.org/10.4213/mzm10818

Использование Общероссийского математического портала Math-Net.Ru подразумевает, что вы прочитали и согласны с пользовательским соглашением http://www . mathnet.ru/rus/agreement

Параметры загрузки:

IP : 54.224 .135 .184

26 апреля 2023 г., 15:54:46

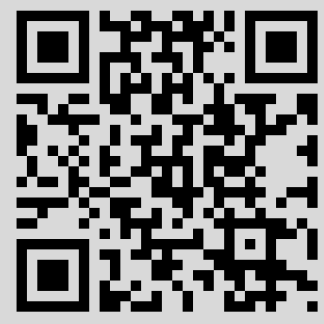




\section{К одной теореме Ловаса о хроматическом числе сферы}

\section{А. М. Райгородский}

1. Введение. В 1981 г. П. Эрдеш ввел в рассмотрение хроматическое число сферы, т.е. величину $\chi\left(S_{r}^{n-1}\right)$, равную минимальному количеству цветов, в которые можно так покрасить все точки на $(n-1)$-мерной сфере $S_{r}^{n-1}$ радиуса $r$ в $\mathbb{R}^{n}$, чтобы между точками одного цвета не было расстояния 1 (расстояние между точками измеряется в объемлющем евклидовом пространстве $\left.\mathbb{R}^{n}\right)$. Легко видеть, что

$$
\chi\left(S_{r}^{n-1}\right)= \begin{cases}1, & r<1 / 2, \\ 2, & r=1 / 2 .\end{cases}
$$

Эрдеш высказал гипотезу, что уже при любом $r>1 / 2$ хроматическое число сферы стремится к бесконечности. Ловас в 1983 г. доказал (см. [1]) даже более сильное утверждение.

Теорема 1 (Ловас). Для любого $r>1 / 2$ и любого $n$ выполена оченка

$$
\chi\left(S_{r}^{n-1}\right) \geqslant n .
$$

Доказательство теоремы 1 довольно нетривиальное: в нем используется одна модификация топологического метода в комбинаторике (см. [2]). В настоящей заметке мы приведем и обсудим совсем короткое доказательство следующего чуть более слабого результата.

Теорема 2. Пусть $\omega$-любая функиия натурального аргумента $n$, которая стремится $\kappa$ бесконечности при $n \rightarrow \infty$. Пусть $r$ - любая функиия натурального аргумента $n$, которая при каждом $n$ не меньше величины $1 / 2+\omega(n) / n$. Тогда

$$
\chi\left(S_{r(n)}^{n-1}\right) \rightarrow \infty \quad \text { npu } \quad n \rightarrow \infty .
$$

Доказательство теоремы 2 мы дадим в следующем пункте. В п. 3 мы обсудим ряд вопросов, которые возникают в связи с полученным результатом.

2. Доказательство теоремы 2. Будем считать, что $\omega=o(n)$. Это не ограничит общности, поскольку ясно, что

$$
\chi\left(S_{r}^{n-1}\right) \geqslant \chi\left(S_{r^{\prime}}^{n-2}\right)
$$

при любых $r, r^{\prime}$, удовлетворяющих неравенству $r>r^{\prime}$. По той же причине будем считать, что число $n$ четное.

Рассмотрим множество точек

$$
V_{n}=\left\{\mathbf{x}=\left(x_{1}, \ldots, x_{n}\right): x_{i} \in\{0,1\}, x_{1}+\cdots+x_{n}=\frac{n}{2}\right\} .
$$

Заметим, что оно лежит на сфере радиуса $\sqrt{n / 4}$.

Пусть $\delta$ - некоторая положительная целочисленная функция аргумента $n$, которая бесконечно мала по отношению к $n$, которая, однако, стремится к бесконечности при $n \rightarrow \infty$ и которую мы подберем чуть позже в зависимости от нашей функции $\omega$. Построим граф $G_{n}=\left(V_{n}, E_{n}\right)$, соединяя две точки $\mathbf{x}, \mathbf{y} \in V_{n}$ ребром тогда и только тогда, когда скалярное произведение $(\mathbf{x}, \mathbf{y})$ равно $\delta(n)$ или, что то же самое, евклидово расстояние между $\mathbf{x}$ и $\mathbf{y}$ равно $\sqrt{n-2 \delta(n)}$. Если отнормировать все точки из $V_{n}$ на $\sqrt{n-2 \delta(n)}$, то длина каждого ребра нового графа $G_{n}^{\prime}=\left(V_{n}^{\prime}, E_{n}^{\prime}\right)$ окажется равной 1 и вершины этого графа будут лежать

Работа выполнена при финансовой поддержке Российского фонда фундаментальных исследований (грант № 15-01-03530), гранта Президента РФ № МД-6008.2015.1 и программы "Ведущие научные школы" (грант № НШ-2964.2014.1).

DOI: $10.4213 / \mathrm{mzm} 10818$ 
на сфере, радиус $r^{\prime}(n)$ которой имеет величину $1 / 2+\Theta(\delta(n) / n)$. Разумеется, не составляет труда так выбрать $\delta$, чтобы величина $r^{\prime}$ была при каждом $n$ меньше величины $r$ из формулировки теоремы 2. Для завершения доказательства достаточно, стало быть, убедиться в том, что хроматическое число $\chi\left(G_{n}^{\prime}\right)$ графа $G_{n}^{\prime}$ стремится к бесконечности.

Выберем в графе $G_{n}$ индуцированный подграф $H_{n}$, вершины которого - это все возможные точки $\mathbf{x} \in V_{n}$ с координатами

$$
x_{1}=x_{2}=\cdots=x_{\delta(n)}=1 .
$$

Очевидно, граф $H_{n}$ изоморфен графу, вершины которого суть $(n / 2-\delta(n))$-элементные подмножества множества мощности $n-\delta(n)$, а ребра - пары подмножеств, имеющих пустое пересечение. Это кнезеровский граф $K G_{n-\delta(n), n / 2-\delta(n)}$, и хорошо известно, что его хроматическое число равно (см. [2])

$$
(n-\delta(n))-2\left(\frac{n}{2}-\delta(n)\right)+2=\delta(n)+2 \rightarrow \infty, \quad n \rightarrow \infty .
$$

Итак,

$$
\chi\left(G_{n}^{\prime}\right)=\chi\left(G_{n}\right) \geqslant \chi\left(H_{n}\right)=\chi\left(K G_{n-\delta(n), n / 2-\delta(n)}\right) \rightarrow \infty, \quad n \rightarrow \infty,
$$

и теорема 2 доказана.

3. Комментарии. Как видно, в доказательстве теоремы 2 есть огромный запас прочности, ведь фактически в нем мы работаем не с евклидовой сферой, но со сферой в хэмминговом пространстве. Вернее, речь идет о графе с вершинами в булевом кубе. Более общий класс графов такого вида устроен следующим образом:

$$
\begin{aligned}
V_{n}(k(n))=\left\{\mathbf{x}=\left(x_{1}, \ldots, x_{n}\right): x_{i} \in\{0,1\}, x_{1}+\cdots+x_{n}=k(n)\right\}, \\
\\
E_{n}(k(n), t(n))=\{\{\mathbf{x}, \mathbf{y}\}:(\mathbf{x}, \mathbf{y})=t(n)\} .
\end{aligned}
$$

Графы

$$
G_{n}(k(n), t(n))=\left(V_{n}(k(n)), E_{n}(k(n), t(n))\right)
$$

были использованы в работах [3], [4], где было доказано, что для любого фиксированного $r>1 / 2$ хроматическое число $\chi\left(S_{r(n)}^{n-1}\right)$ растет экспонециально по $n$ и что при $r \geqslant$ $1 / 2+c / n^{0.475}$ с достаточно большим $c>0$ выполнена оценка $\chi\left(S_{r(n)}^{n-1}\right)>n+2$, вопреки одному ложному утверждению Ловаса, сделанному все в той же работе [1]. Любопытно, что метод, примененный в работах [3], [4] для обоснования нижних оценок хроматических чисел графов $G_{n}(k(n), t(n))$, - а именно, линейно-алгебраический метод (см. [5], [6]) - не позволяет получать растущих оценок для графов из доказательства теоремы 2. С другой стороны, оценка, которой мы воспользовались здесь, кажется весьма грубой. Посему значительный интерес представляет отыскание хроматических чисел графов вида $G_{n}(n / 2, \delta(n))$ при медленно растущих $\delta(n)$.

\section{СПИСОК ЦИТИРОВАННОЙ ЛИТЕРАТУРЫ}

[1] L. Lovász, Acta Sci. Math. (Szeged), 45 (1983), 317-323. [2] J. Matoušek, Using the Borsuk-Ulam Theorem, Universitext, Springer-Verlag, Berlin, 2003. [3] А. М. Райгородский, Докл. РАН, 432:2 (2010), 174-177. [4] A. M. Raigorodskii, Combinatorica, 32:1 (2012), 111-123. [5] А. М. Райгородский, УМH, 56:1 (2001), 107-146. [6] А. М. Райгородский, Линейно-алгебраический метод в комбинаторике, МЦНМО, М., 2007.

\section{А. М. Райгородский}

Поступило

Московский государственный

09.12.2014

университет имени М.В.Ломоносова;

Московский физико-технический

институт (государственный университет),

г. Долгопрудный Московской обл.

E-mail: mraigor@yandex.ru 